\section{Acute Gouty Arthritis After Taking Sildenafil: An Old Disease with a New Etiology}

\section{To the Editor:}

Sildenafil citrate (Viagra), a selective inhibitor of cyclic guanosine monophosphate (cGMP)-specific phosphodiesterase type 5, is an effective treatment for male erectile dysfunction. By enhancement of the effect of nitric oxide and cGMP, it leads to smooth-muscle relaxation and inflow of blood to the corpus cavernosum ${ }^{1}$. Sildenafil has suitable pharmacodynamic and pharmacokinetic properties for use as an oral therapeutic drug for erectile dysfunction ${ }^{2}$. Numerous adverse reactions to sildenafil have been reported, including headache, facial flush, dyspepsia, nasal stuffiness, and conjunctival suffusion. However, there have been no reports that sildenafil is associated with acute gout. We describe a 41-year-old man who developed gouty arthritis after taking sildenafil. There was a temporal relation between the attacks of gouty arthritis and administration of sildenafil. Symptoms then subsided after the withdrawal of sildenafil. The symptoms did recur when he took sildenafil again (positive rechallenge test). Recognizing the possible association and prompt management will prevent further unnecessary attacks.

A previously healthy 41-year-old man with no significant medical history presented to our outpatient department (first visit) with sudden onset of severe exquisite pain and swelling at the metatarsophalangeal (MTP) joint of the right hallux. He had had 6 or 7 similar attacks in the past 5 months. He had visited 3 primary care physicians, who prescribed "pain killers" for his acute symptoms. He specifically denied illicit drug use. There was no contributory family history or drug and food allergy history. Examination showed tenderness, swelling, and redness over the MTP joint. Laboratory data revealed normal findings except his serum level of uric acid was $9.0 \mathrm{mg} / \mathrm{dl}$ (reference value $<7.0$ ). Based on the diagnostic criteria of the American Rheumatism Association ${ }^{3}$, he was diagnosed as having acute gouty arthritis at second visit. By taking sulindac and colchicine, followed by uric acid-lowering agents (benzbromarone), he had been able to have $2-3$ weeks symptom-free. Then he presented (third visit) at the outpatient department and asked to see the attending physician (CHL) privately. He stated he had had another attack of acute arthritis, which he believed was because he had taken $50 \mathrm{mg}$ sildenafil one night before. $\mathrm{He}$ also recalled that there had been a temporal relation between the use of sildenafil and the previous episodes of attacks. He was advised not to take sildenafil until we consulted other specialists.

An extensive literature review failed to find any report relating sildenafil use and gouty arthritis. Confirmation of acute arthritis of gout related to sildenafil by rechallenge test would be reliable but unjustifiable ${ }^{4}$. Three weeks later (fourth visit), he again developed painful swelling at the same location one day after taking sildenafil $50 \mathrm{mg}$. Synovial fluid analysis confirmed the presence of monosodium urate crystals. After treatment, he has been symptom-free with discontinuation of sildenafil for more than 2 months since that episode. In December 2007 (fifth visit), a followup examination was unremarkable and his uric acid level was $4.6 \mathrm{mg} / \mathrm{dl}$ without use of urate-lowering agents.

It is tempting to speculate that sildenafil induced gout episodes in this case because this has not been documented in any report. In addition, gout flares are often observed when urate-lowering therapy has been started or stopped. The temporal sequences in this case do not absolutely preclude this as an explanation of the gout flares. However, the temporal association between administration of sildenafil and episodes of acute gouty arthritis implies a causal relation. The recurrence of acute arthritis after taking sildenafil (rechallenge test) also suggests a causal relation. Gouty arthritis is listed as one of many side effects (Viagara package insert; Pfizer Inc.), so it must have been detected during the phase III clinical trial. In addition, an unpublished safety surveillance and reporting from Pfizer Inc. describes 7 adverse events with gout attacks during use of sildenafil ${ }^{5}$.

Sildenafil has been widely prescribed; in the absence of evidence from reports in the literature it is imperative to be aware of this problem. We conclude that sildenafil can induce acute gouty arthritis, and any physician encountering a case with acute arthritis of primary gout will need to suspect the possibility of this etiology. The lack of this awareness may result in delay recognizing the etiology and providing prompt management.

WEI-LIANG CHEN, MD, Department of Family Medicine and Community Health; HONG-I CHEN, MD, PhD, Division of Urology; CHING-HUI LOH, MD, PhD, Department of Family Medicine and Community Health, Tri-Service General Hospital, National Defense Medical Center, Number 325, Section 2, Cheng-Kung Road, Neihu 114, Taipei, Taiwan. Address reprint requests to Dr. Loh;

E-mail: twdoc@ndmctsgh.edu.tw

\section{REFERENCES}

1. Boolell M, Gepi-Attee S, Gingell J, Allen M. Sildenafil, a novel effective treatment for male erectile dysfunction. Br J Urol 1996;78:257-61.

2. Boolell M, Allen MJ, Ballard SA, et al. Sildenafil: an orally active type 5 cyclic GMP-specific phosphodiesterase inhibitor for the treatment of penile erectile dysfunction. Int J Impot Res 1996; $8: 47-52$

3. Wallace SL, Robinson H, Masi AT, Decker JL, McCarty DJ, Yu TF Preliminary criteria for the classification of the acute arthritis of primary gout. Arthritis Rheum 1977;20:895-900.

4. Loh CH, Cang YW, Liou SH, Chang JH, Chen HI. Case report: hexachloroethane smoke inhalation: a rare cause of severe hepatic injuries. Environ Health Perspect 2006;114:763-5.

5. D'Amato S, Hassett J. Summary bridging report: Sildenafil, safety surveillance and reporting. New York: Pfizer Inc.; 2006.

J Rheumatol 2009;36:1; doi:10.3899/jrheum.080572 